\title{
"Brinquedos observados no ambiente domiciliar de lactentes: uma comparação entre meninos e meninas"
}

\author{
"Toys observed in the home environment of infants: \\ a comparison between boys and girls"
}

RESUMo Introdução: Brinquedos são fonte de estímulo ao desenvolvimento infantil na medida em que proporcionam affordances (possibilidades de ação motora). Embora diversos estudos investiguem affordances no ambiente domiciliar, nenhum observou se há diferenças entre lactentes meninos e meninas.

Objetivo: Avaliar os brinquedos disponíveis no ambiente domiciliar de bebês do sexo masculino e feminino.

Método: Estudo observacional e transversal, no qual participaram 30 lactentes, 15 do sexo feminino, idade média de $7( \pm 1)$ meses. Para o levantamento dos brinquedos no ambiente domiciliar, foi utilizado o questionário Affordances no Ambiente Domiciliar para o Desenvolvimento Motor - Escala Bebê (AHEMD-IS), dimensões brinquedos de motricidade fina e grossa. Para caracterização do grupo, foram coletados dados do nascimento do bebê e da condição econômica da família. Para a análise dos dados, foi utilizado o teste exato de Fisher.

Resultados: Os resultados apontam para baixa quantidade de brinquedos disponíveis no ambiente domiciliar dos lactentes. Não foi observada associação significativa na disponibilidade de brinquedos entre meninas e meninos.

Conclusão: Considerando que os brinquedos oferecem oportunidades de ação para o desenvolvimento motor dos bebês, o estudo mostrou restrição de affordances (possibilidades de ação motora) relacionadas a essa dimensão do ambiente, o que pode repercutir no desempenho motor dos lactentes.

Palavras chaves: Lactente. Brinquedo. Desempenho perceptual-motor. Domicílio.

Bianca Alves Almozara BONTORIM $^{\mathrm{I}}$

Audrei Fortunato MiQuelote ${ }^{1}$ Cassiana do Amaral Coppola ANDREa Baraldi CunHa ${ }^{\text {II }}$ Gabriella Padovani ${ }^{1}$ VANessa Araújo Silva ${ }^{1}$ Denise Castilho Cabrera SANTOS ${ }^{\mathrm{I}}$

${ }^{1}$ Universidade Metodista de

Piracicaba (UNIMEP), Piracicaba/

SP - Brasil.

"Universidade Federal de São Carlos (UFSCAR), São Carlos/SP - Brasil.

ABstract Introduction: Toys are a source of stimulus for child development to the extent that they provide affordances (possibilities of motor action). Although several studies investigate affordances in the home environment, none has noted whether there are differences between boys and girls infants.

Objective: To evaluate the available toys in the home environment of male and female infants.

Method: Observational and transversal study, in which 30 infants participated, 15 female, mean age of $7( \pm 1)$ months. To assess the quantity of the toys in the home environment, we used the dimensions of gross-motor toys and fine-motor toys of the Affordances in the Home Environment for Motor Development - Infant Scale (AHEMD-IS). To characterize the group, it was collected the data of infants' birth and the socioeconomic status of the family. Fisher's exact test was used to analyze the data. Results: The results point to the low quantity of toys available in the home environment of infants. No significant association was found on the availability of toys between girls and boys. Conclusion: Considering that toys offer opportunities for action for the motor development of infants, the study showed a restriction of affordances related to this dimension of the environment, which can affect the motor performance of infants.

Key-words: Infant. Playthings. Psychomotor performance. Housing. 


\section{INTRODUÇÃo}

Gabbard $^{1}$ define o desenvolvimento motor como um processo de mudança resultante da interação da hereditariedade com o ambiente, destacando que, para entender o comportamento motor em qualquer fase da vida, deve-se valorizar, entre outras coisas, a história, a cultura e as oportunidades de prática de cada indivíduo.

A relação recíproca entre indivíduo-ambiente foi proposta por Gibson, como um pressuposto da teoria Ecológica (Affordances).$^{2-5}$ Gibson ressalta a relação entre percepção e ação, propondo então o que ele denominou de affordances, que são "oportunidades que oferecem um potencial individual para ação motora, consequentemente aprendizado e desenvolvimento de uma habilidade ou uma parte do sistema biológico".,2,4,-8

Miquelote et al. ${ }^{9}$ analisaram a influência de affordances no ambiente domiciliar para o desenvolvimento motor de bebês e possíveis efeitos nas habilidades motoras e cognitivas. Os resultados mostraram que as affordances motoras podem impactar positivamente as habilidades motoras ao longo do tempo e possivelmente a cognição do bebê. Diante disso, pode-se considerar que um ambiente positivo (estimulante, rico em affordances) facilita o desenvolvimento típico do lactente, pois permite a interação e a exploração com o meio. Enquanto, um ambiente desfavorável pode limitar o desenvolvimento e as possibilidades de aprendizado da criança. ${ }^{3,10-11}$

Caçola, Gabbard, Santos e Batistela ${ }^{12}$ encontraram associação significativa entre affordances motoras no ambiente domiciliar, especificamente brinquedos que estimulem a motricidade fina e grossa, e os resultados do desempenho motor de lactentes. Os autores concluíram que a disponibilidade de brinquedos apropriados à idade do bebê pode predizer, em parte, seu comportamento motor. Estudo de Correr et al. ${ }^{3}$ mostrou que, para lactentes entre três e 11 meses, a disponibilidade de brinquedos apropriados à idade tende a estimular seu uso gerando oportunidades de ação (affordances). Dessa forma, entende-se que brinquedos apropriados à idade, disponíveis no ambiente domiciliar, são fonte de estímulo ao desenvolvimento infantil na medida em que proporcionam affordances (possibilidades de ação motora).

Ginsburg et al., ${ }^{13} \mathrm{em}$ um artigo de revisão sobre a importância do brincar na promoção do desenvolvimento saudável da criança, trazem como recomendação aos pediatras, que estes devem enfatizar os benefícios dos "brinquedos verdadeiros", como blocos e bonecos, com os quais as crianças utilizam plenamente a sua imaginação, em detrimento de brinquedos passivos que exigem imaginação limitada.

Na mesma linha de argumentação, Wanderlind et al., ${ }^{14} \mathrm{em}$ um estudo observacional, apontam que as diferenças entre os meninos e meninas no brincar estão relacionadas a múltiplos fatores que interagem ao longo do processo de desenvolvimento, sendo o ambiente em que a criança se insere o que mais influencia o comportamento e diferentes formas de desempenhar as atividades. Apesar das brincadeiras e brinquedos serem relatados como coadjuvantes no desenvolvimento infantil, a carência de estudos com diferentes sexos e ambientes é notória na literatura. ${ }^{13}$ 
Neste estudo, serão exploradas questões referentes às affordances (oportunidades de ação) oferecidas pelo ambiente domiciliar de bebês do sexo masculino ou feminino, com foco para a presença de brinquedos. Desse modo, o objetivo deste estudo foi avaliar os brinquedos disponíveis no ambiente domiciliar e analisar possível associação com o sexo dos lactentes. Nossa hipótese é que os brinquedos disponíveis no ambiente domiciliar são escassos devido à falta de recursos socioeconômicos, não havendo diferenças entre tipo e quantidade de brinquedos entre bebês do sexo feminino e masculino.

\section{MÉTODO}

Trata-se de estudo observacional, com delineamento transversal, no qual famílias foram avaliadas quanto às características do ambiente familiar relevantes para o desenvolvimento de seus bebês, considerando as oportunidades para o desenvolvimento motor referentes ao tipo e quantidade de brinquedos. Esse projeto foi aprovado pelo Comitê de Ética em Pesquisa da UNIMEP, CEP/UNIMEP nº. 81/2015.

A amostra foi composta por 30 lactentes, sendo 15 do sexo feminino, com idade entre 6-8 meses, participantes da Pastoral da Criança do município de Piracicaba-SP e cujos pais assinaram Termo de Consentimento Livre e Esclarecido.

Após o convite para a participação no estudo e permissão da família por meio do Termo de Consentimento Livre e Esclarecido, foi utilizado o Critério de Classificação Econômica do Brasil da Associação Nacional de Empresas de Pesquisa (ABEP 2015). ${ }^{15}$ $\mathrm{O}$ questionário utiliza questões referentes a bens de consumo, escolaridade do chefe da família e serviços públicos (água e pavimentação) para a classificação econômica da família.

Além disso, foi realizada uma cópia da Caderneta de Saúde da Criança, obtendo-se informações referentes aos indicadores de risco e condições de saúde dos lactentes ao nascimento (índice de APGAR no $1^{\circ}$. e $5^{\circ}$. minutos, idade gestacional e peso ao nascer).

Em seguida, os pais ou responsáveis responderam ao questionário Affordances no Ambiente Domiciliar para o Desenvolvimento Motor - Escala Bebê (AHEMD-IS)., ${ }^{2,6}$ Trata-se de questionário brasileiro, que avalia as affordances do ambiente domiciliar de lactentes de 3-18 meses de idade, composto das dimensões espaço físico, variedade de estimulação, brinquedos que fornecem oportunidades para o desenvolvimento motor grosso $(\mathrm{MG})$ e brinquedos que fornecem oportunidades para o desenvolvimento motor fino (MF). ${ }^{2,6}$ Ao final, o ambiente e suas dimensões pode ser classificado em: "Menos que o adequado", "Moderadamente adequado", "Adequado" e "Excelente" quanto às affordances que oportunizam ao lactente.

A seção brinquedos do AHEMD-IS contém questões específicas para diferentes idades, sendo 11 (6 MG e $5 \mathrm{MF}$ ) direcionadas para lactentes até 11 meses, acrescidas de mais 9 para lactentes até 18 meses. Tendo em vista que os lactentes incluídos no estudo tinham entre 6-8 meses de vida, a avaliação da dimensão brinquedos foi realizada até a questão de número 26 do questionário AHEMD-IS. Cada uma das 20 questões referentes aos brinquedos tem descrição e ilustração (exemplo), seguidas da pergun- 
ta "Quantos destes brinquedos você utiliza para brincar com seu bebê em casa?", com três níveis de respostas "Nenhum, Um dois, Três ou mais” (Figura 1).

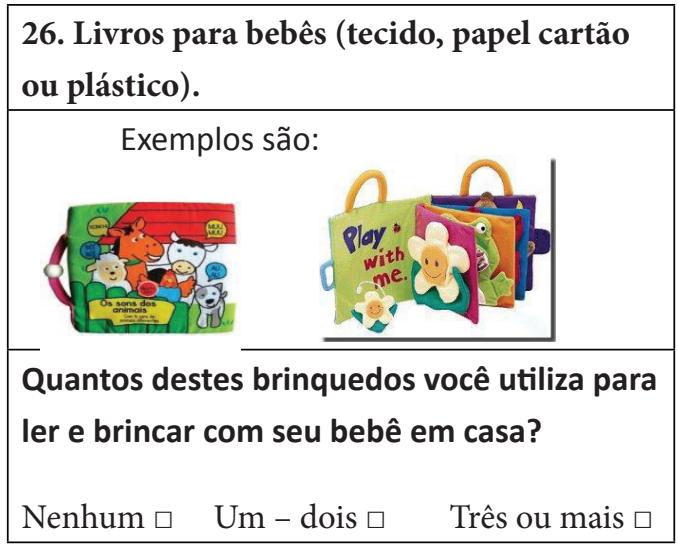

Figura 1: exemplo de questão da seção brinquedos do questionário AHEMD-IS.

Para a análise dos dados, foi utilizada a estatística descritiva e o teste exato de Fisher para dados categóricos para investigar a associação entre affordances que o ambiente oferece para o desenvolvimento motor, com ênfase nos tipos e quantidades de brinquedos, e o sexo dos lactentes. Para a análise das questões referentes à quantidade de brinquedos, as respostas foram dicotomizadas em "Nenhum" ou "Um/ou mais" (inclui as respostas: Um-dois e Três ou mais). De modo semelhante, a classificação das dimensões brinquedos de motricidade grossa e de motricidade fina foi dicotomizada em Abaixo do adequado (inclui as classes: Menos que o adequado e Moderadamente adequado) ou Adequado ou acima (inclui as classes: Adequado e Excelente). O nível de significância considerado foi de $5 \%$.

\section{RESUltados}

A idade média dos 30 participantes do estudo foi de $7( \pm 1)$ meses, sendo $15(50 \%)$ do sexo feminino. Os lactentes nasceram com boas condições de saúde relacionadas ao peso (29/30 lactentes com peso ao nascer $\geq 2500 \mathrm{~g})$, duração da gestação (29/30 lactentes com idade gestacional $\geq 37$ semanas) e todos 30/30 tiveram índice de Apgar $\geq$ a 8 no $1^{\circ}$. e $\geq$ a 9 no $5^{\circ}$. minutos de vida. Já os dados obtidos por meio do questionário ABEP 2015 revelaram que as classes econômicas $\mathrm{C} 1, \mathrm{C} 2 \mathrm{e} \mathrm{D} / \mathrm{E}$ foram as predominantes (23/30 ou $77 \%)$ nas famílias participantes do estudo. As demais famílias, $07 / 30$ ou $23 \%$, enquadraram-se nas classes B1, B2 ou A.

As Tabelas 1 e 2 mostram os dados referentes às questões sobre brinquedos que estimulam predominantemente a motricidade grossa (16 a 21) e que estimulam predominantemente a motricidade fina (22 a 26) presentes no ambiente domiciliar dos lactentes.

Os dados na Tabela 1 mostram que em quase todas as questões respondidas a maioria das meninas e dos meninos não possuía qualquer dos brinquedos de motricidade grossa pesquisados (questões 16 e 18 a 21). Apenas a questão número 17 (bonecos de pelúcia, brinquedos, emborrachados, de tecidos ou de brincar na água) mostrou que $11 / 15$ meninas e 11/15 meninos tinham um ou mais brinquedos desse tipo. Logo, pode-se observar que os bonecos de pelúcias, emborrachados ou de diferentes tecidos são os brinquedos de motricidade grossa mais encontrados no âmbito familiar pesquisado. 
Tabela 1: Brinquedos de motricidade grossa descritos em frequência de respostas das famílias, divididos pelo sexo dos lactentes.

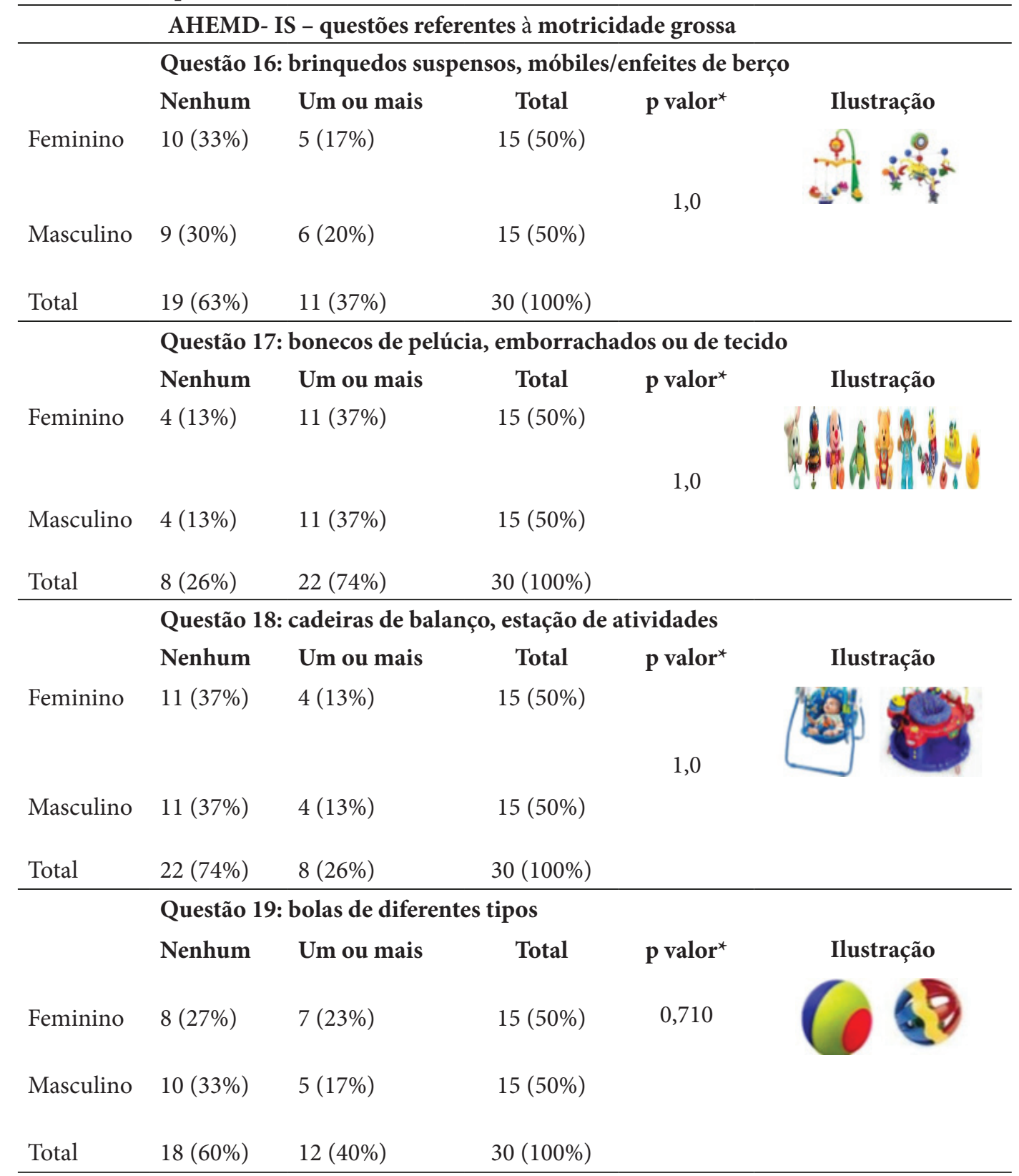




\begin{tabular}{|c|c|c|c|c|c|}
\hline \multirow[b]{3}{*}{ Feminino } & \multicolumn{5}{|c|}{ Questão 20: colchonete, tapete emborrachado } \\
\hline & \multirow{2}{*}{$\begin{array}{l}\text { Nenhum } \\
11(37 \%)\end{array}$} & \multirow{2}{*}{$\begin{array}{l}\text { Um ou mais } \\
4(13 \%)\end{array}$} & \multirow{2}{*}{$\begin{array}{c}\text { Total } \\
15(50 \%)\end{array}$} & \multirow[t]{2}{*}{ p valor* } & \multirow[t]{2}{*}{ Ilustração } \\
\hline & & & & & \\
\hline Masculino & $10(33 \%)$ & $5(17 \%)$ & $15(50 \%)$ & & \\
\hline \multirow[t]{3}{*}{ Total } & $21(70 \%)$ & $9(30 \%)$ & $30(100 \%)$ & & \\
\hline & \multicolumn{5}{|c|}{ Questão 21: Materiais musicais } \\
\hline & Nenhum & Um ou mais & Total & p valor* & Ilustração \\
\hline Feminino & $8(27 \%)$ & $7(23 \%)$ & $15(50 \%)$ & & \\
\hline Masculino & 8 (270) & 7 (230\%) & $15(50 \%)$ & & \\
\hline Masculino & $8(27 \%)$ & $7(23 \%)$ & $15(50 \%)$ & & \\
\hline Total & $16(54 \%)$ & $14(46 \%)$ & $30(100 \%)$ & & \\
\hline
\end{tabular}

^Teste Exato de Fisher

A Tabela 2 mostra que em apenas uma questão relacionada a brinquedos de motricidade fina (questão 22 - brinquedos manipuláveis tipo chocalhos e mordedores) $15 / 15$ meninas e 12/15 meninos tinham um ou mais brinquedos desse tipo. Nas demais questões, a maioria dos lactentes não tinha qualquer dos brinquedos de motricidade fina pesquisados. Portanto, os brinquedos de motricidade fina observados entre os lac- tentes avaliados foram apenas os chocalhos e mordedores.

Considerando que os brinquedos oferecem oportunidades de ação motora para os lactentes, o estudo revela uma restrição de affordances relacionadas aos brinquedos tanto de motricidade grossa quanto de motricidade fina, o que pode causar impactos no desenvolvimento motor desses lactentes. 
Tabela 2: Brinquedos de motricidade fina - dados descritivos em frequência de respostas das famílias, divididos pelo sexo.

\begin{tabular}{|c|c|c|c|c|c|}
\hline \multicolumn{6}{|c|}{ AHEMD- IS - questões referentes à motricidade fina } \\
\hline & \multicolumn{5}{|c|}{ Questão 22: brinquedos manipuláveis (chocalho, mordedor) } \\
\hline & Nenhum & $\begin{array}{c}\text { Um ou } \\
\text { mais }\end{array}$ & Total & p valor ${ }^{*}$ & Ilustração \\
\hline Feminino & $0(0 \%)$ & $15(50 \%)$ & $15(50 \%)$ & \multirow{2}{*}{0,224} & \\
\hline Masculino & $3(10 \%)$ & $12(40 \%)$ & $15(50 \%)$ & & \\
\hline \multirow[t]{3}{*}{ Total } & $3(10 \%)$ & $27(90 \%)$ & $30(100 \%)$ & & \\
\hline & \multicolumn{4}{|c|}{ Questão 23: carros, trens, animais } & \multirow{3}{*}{ Ilustração } \\
\hline & Nenhum & $\begin{array}{c}\text { Um ou } \\
\text { mais }\end{array}$ & Total & p valor & \\
\hline Feminino & $13(43 \%)$ & $2(7 \%)$ & $15(50 \%)$ & \multirow{2}{*}{0,651} & \\
\hline Masculino & $11(37 \%)$ & $4(13 \%)$ & $15(50 \%)$ & & \multirow[b]{4}{*}{ Ilustração } \\
\hline \multirow[t]{2}{*}{ Total } & $24(80 \%)$ & $6(20 \%)$ & $30(100 \%)$ & & \\
\hline \multicolumn{5}{|c|}{ Questão 24: brinquedos de apertar, acionar } & \\
\hline & Nenhum & $\begin{array}{c}\text { Um ou } \\
\text { mais }\end{array}$ & Total & p valor ${ }^{*}$ & \\
\hline Feminino & $11(37 \%)$ & $4(13 \%)$ & $15(50 \%)$ & \multirow{2}{*}{0,598} & \multirow[b]{5}{*}{ Ilustração } \\
\hline Masculino & $11(37 \%)$ & $4(13 \%)$ & $15(50 \%)$ & & \\
\hline \multirow[t]{3}{*}{ Total } & $22(74 \%)$ & $8(26 \%)$ & $30(100 \%)$ & & \\
\hline & \multicolumn{4}{|c|}{ Questão 25: blocos } & \\
\hline & Nenhum & $\begin{array}{c}\text { Um ou } \\
\text { mais }\end{array}$ & Total & p valor ${ }^{*}$ & \\
\hline Feminino & $13(43 \%)$ & $2(7 \%)$ & $15(50 \%)$ & \multirow{2}{*}{0,483} & \\
\hline Masculino & $15(50 \%)$ & $0(0 \%)$ & $15(50 \%)$ & & \\
\hline \multirow[t]{3}{*}{ Total } & $28(93 \%)$ & $2(7 \%)$ & $30(100 \%)$ & & \\
\hline & \multicolumn{4}{|c|}{ Questão 26: livros para bebês } & \\
\hline & Nenhum & $\begin{array}{l}\text { Um ou } \\
\text { mais }\end{array}$ & Total & $\underset{\text { valor }^{*}}{\mathbf{p}}$ & Ilustração \\
\hline Feminino & $12(40 \%)$ & $3(10 \%)$ & $15(50 \%)$ & \multirow{3}{*}{1,0} & 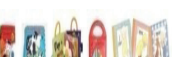 \\
\hline Masculino & $13(43 \%)$ & $2(7 \%)$ & $15(50 \%)$ & & 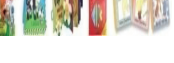 \\
\hline Total & $25(83 \%)$ & $5(17 \%)$ & $30(100 \%)$ & & \\
\hline
\end{tabular}


Tabela 3: Disponibilidade de brinquedos no ambiente domiciliar de lactentes meninos e meninas.

\begin{tabular}{ccllc}
\hline $\begin{array}{c}\text { Brinquedos Motricidade } \\
\text { Grossa }\end{array}$ & $\begin{array}{c}\text { Abaixo do } \\
\text { adequado }\end{array}$ & $\begin{array}{c}\text { Adequado ou } \\
\text { acima }\end{array}$ & Total & p valor* \\
\hline Meninos & $13(43 \%)$ & $2(7 \%)$ & $15(50 \%)$ & 1,00 \\
Meninas & $12(40 \%)$ & $3(10 \%)$ & $15(50 \%)$ & \\
Total & $25(83 \%)$ & $5(17 \%)$ & $30(100 \%)$ & \\
\hline Brinquedos Motricidade & Abaixo do & $\begin{array}{c}\text { Adequado ou } \\
\text { acima }\end{array}$ & Total & p valor \\
Fina & adequado & $3(10 \%)$ & $15(50 \%)$ & 1,00 \\
Meninos & $12(40 \%)$ & $2(7 \%)$ & $15(50 \%)$ & \\
Meninas & $13(43 \%)$ & $5(17 \%)$ & $30(100 \%)$ & \\
Total & $25(83 \%)$ & &
\end{tabular}

*Teste Exato de Fisher

Como pode ser observado na Tabela 3, não foi identificado associação significativa entre as oportunidades de ação motora (affordances) disponibilizadas no ambiente domiciliar e o lactente ser menina ou menino.

\section{Discuss Ão}

O estudo foi conduzido com o objetivo de avaliar os brinquedos disponíveis no ambiente domiciliar de lactentes e analisar se a disponibilidade dessas affordances tinha associação com o sexo dos lactentes.

Analisando as dimensões Brinquedos de Motricidade Grossa e Brinquedos de Motricidade Fina, observou-se que tanto as meninas quanto os meninos tinham poucos brinquedos, o que é uma situação negativa de estimulação. Os únicos brinquedos identificados, para a maioria dos meninos e meninas, foram bonecos de pelúcia, emborrachados, de tecido e chocalhos e mordedores.

Estudos demonstram que o brincar é uma das atividades que a criança mais gosta e uma das mais importantes para o seu desenvolvimento integral., 17-18 $\mathrm{O}$ brincar é uma atividade natural da infância, a qual produz inúmeras oportunidades para que a criança aprenda e se desenvolva física, mental e socialmente. ${ }^{7,17-18}$

Ainda que o brincar não dependa de a criança ter ou não brinquedos, eles são os elementos essenciais para proporcionar o brincar, uma vez que os brinquedos dão origem às brincadeiras e proporcionam experiências variadas. ${ }^{13}$ Em um estudo com crianças com Paralisia Cerebral, Braga e Graciani ${ }^{19}$ apontam a importância do brincar e da utilização de brinquedos e recursos lúdicos, destacando que os estímulos e a motivação gerados pelos mesmos despertam na criança a vontade de realizar a atividade proposta, levando a novas descobertas e exploração do seu próprio corpo. O amplo uso dos brinquedos como fonte de estimulação do desenvolvimento foi apontado por Siaulys $^{20}$ em material publicado pelo $\mathrm{Mi}$ nistério da Educação. O brincar auxilia nos processos do convívio e socialização fazendo que a criança interaja com o meio, assim, há desenvolvimento intelectual, sensório motor, da criatividade, dos valores morais, 
além do valor terapêutico, possibilitando que a criança vivencie diferentes emoções. ${ }^{17,18,21}$ Além disso, brincar proporciona movimentos, os quais expressam emoções, sentimentos, gestos e posturas corporais. ${ }^{7,17}$

Utilizar brinquedos adequados para a idade representa oportunidades de ação ou affordances à criança. ${ }^{3}$ Gibson expõe que o significado e a utilidade do ambiente estão diretamente ligados ao indivíduo, e a interação ocorrida entre ambos, propiciando uma relação funcional entre um objeto e um indivíduo com características próprias, inserido em determinado ambiente. ${ }^{3,5,16}$ Então, um ambiente com uma diversidade de brinquedos apropriados à idade do lactente possibilitará a ele oportunidades de percepção e exploração conduzindo à ação motora, ou seja, fornecendo affordances. Para Gibson, não importa a qualidade do objeto em si nem as suas propriedades, mas sim a maneira pela qual o ambiente irá alterar o comportamento e a experiência do indivíduo., ${ }^{5,16}$

Nosso estudo não evidenciou associação entre tipo/quantidade de brinquedos e o sexo dos lactentes. Possivelmente, a baixa condição econômica das famílias levou a um reduzido número e variedade de brinquedos como apontado nos resultados. Outros estudos encontraram associação entre tipo de brinquedo e sexo da criança.

Diversos estudos divergem dos nossos resultados, demonstrando diferenças entre tipo de brinquedos entre meninas e meninos. Um estudo com fotos de brinquedos considerados preferidos pelos bebês de 3 a 18 meses mediu o tempo em que as meninas e os meninos olhavam para cada foto. O estudo revelou que os meninos de 9 meses de idade preferiram as fotos com bolas, blocos e carros, enquanto as meninas de 18 meses não mostraram preferência por qualquer tipo de brinquedo. ${ }^{22}$ Outro estudo realizado em uma creche no Reino Unido, utilizou brinquedos considerados para meninas (boneca, ursinho cor-de-rosa e panela) e para meninos (um carro, ursinho azul, escavadora e uma bola), que foram apresentados a 47 meninas e 50 meninos, divididos em três grupos de acordo com a faixa etária ( 9 a 17 meses, 18 a 23 meses e 24 a 32 meses). O resultado revelou que todos os grupos demonstraram preferências por brinquedos estereotipados para meninos e meninas em cada uma das faixas etárias, concluindo que as diferenças de sexo na preferência por brinquedos aparecem no início do desenvolvimento. ${ }^{23}$ De modo diferente dos autores citados, Wanderlinde et al. ${ }^{15}$ apontam que até os 18 meses as crianças não têm preferências por brinquedos de acordo com o sexo, já aos 3 anos de idade demonstram preferência por brincar com crianças do mesmo sexo e com brinquedos adotados como de meninos e de meninas.

Em nosso estudo, os lactentes eram ainda mais jovens (6-8 meses de idade) do que os que participaram dos estudos citados. ${ }^{15,22,23}$ Dessa forma, a idade do grupo estudado pode ser também uma possível razão para a ausência de associação.

\section{CONSIDERAÇões FINAIS}

De maneira geral, os resultados deste estudo apontam que os lactentes tinham escassos brinquedos, tanto de motricidade grossa quanto de motricidade fina. Não foi encontrada associação entre sexo dos lac- 
tentes e o tipo e a quantidade de brinquedos ou a classificação do ambiente domiciliar quanto às affordances relacionadas aos brinquedos de motricidade grossa e fina.

Considerando que os brinquedos oferecem oportunidades de ação para o de- senvolvimento motor dos bebês, o estudo mostrou restrição de affordances (possibilidades de ação) relacionadas a essas dimensões do ambiente para ambos os sexos, o que pode repercutir no desempenho motor dos lactentes.

\section{REFERÊNCIAS}

1. GABBARD CP. Lifelong Motor Development, 3. ed. Boston: Allyn and Bacon, 2000.

2. CAÇOLA PM, GABBARD C., MONTEBELO MIL, SANTOS DCC. Further Development and Validation of the Affordances in the Home Environment for Motor Development Infant Scale (AHEMD-IS). Physical Therapy. 2015a; 95 (6): 901-923.

3. CORRER MT, OURO MPC, CAÇOLA PM, ALMEIDA TGA, SANTOS DCC. A disponibilidade de brinquedos no ambiente domiciliar representa oportunidades para o desenvolvimento motor de lactentes? Temas sobre Desenvolvimento, 2014; 20: 25-29.

4. COOK AS, WOOLLACOTT MH. Controle motor: teoria e aplicações práticas, 3. ed. Tamboré: Manole, 2010.

5. OLIVEIRA FIS, RODRIGUES ST. Affordances: a relação entre agente e ambiente. Ciências e Cognição. 2006; 9: 120-130.

6. CAÇOLA PM, GABBARD C., MONTEBELO MIL, SANTOS DCC. The new affordances in the home environment for motor development - infant scale (AHEMD-IS): Versions in English and Portuguese languages. Brazilian Journal of Physical Therapy. 2015b; 19 (6): 507-525.

7. FREITAS TCB, GABBARD C., CAÇOLA PM, MONTEBELO MIL, SANTOS DCC. Family socioeconomic status and the provision of motor affordances in the home. Brazilian Journal of Physical Therapy. 2013; 17(4).

8. NOBRE FSS et al. Affordances em Ambientes Domésticos e Desenvolvimento Motor de Pré-Escolares. Pensar a Prática. 2012; 15 (3): 652-668.

9. MIQUELOTE AF, SANTOS DC, CAÇOLA PM, MONTEBELO MI, GABBARD C. Effect of the home environment on motor and cognitive behavior of infants. Infant Behavior and Development. 2012; 35 (3): 329-334.

10. CORSI C., DOS SANTOS MM, MARQUES LAP, ROCHA NACF. Repercussões de Fatores extrínsecos no desempenho motor fino de crianças frequentadoras de creche. Revista Paulista de Pediatria. 2016; 34 (4): 439-446.

11. WILLRICH A., AZEVEDO CCF, FERNANDES J. O. Desenvolvimento motor na infância: influência dos fatores de risco e programas de intervenção. Revista Neurociência. 2009; 17 (1): 51-56.

12. CAÇOLA PM, GABBARD C., SANTOS DCC, BASTISTELA AC. et al. Development of the Affordances in the Home Environment for Motor Development - Infant Scale. Pediatrics International. 2011; 53(6): 820-825.

13. GINSBURG RK. The Importance of Play in Promoting Healthy Child Development and Maintaining Strong Parent-Child Bonds. American Academy of Pediatrics. 2007; 128 (1): 182-191.

14. ASSOCIAÇÃO NACIONAL DE EMPRESAS DE PESQUISA (ABEP). Critério de classificação econômica Brasil. São Paulo: Associação Nacional de Empresas de Pesquisa, 2015.

15. WANDERLIND F. et al. Diferenças de gênero no brincar de crianças pré-escolares e escolares na brinquedoteca. Paidéia. 2006; 16 (34): 263-273. 
16. MIQUELOTE AF. Correlação entre as características do ambiente domiciliar e o desempenho motor e cognitivo de lactentes. Dissertação (Mestrado em Ciências do Movimento Humano) - Universidade Metodista de Piracicaba, Piracicaba, 2011.

17. NELSON FFA, SILVA RL, FIGUEIREDO ZCC. O brincar/jogar como fenômeno transicional na construção da autonomia e da identidade da criança de zero a seis anos. Revista Brasileira Ciência e Esporte. 2006; 27 (2): 75-90.

18. TOLOCKA RE, HORITA KY, OLIVEIRA CBO, COELHO VAC, SANTOS DCC. Como brincar pode auxiliar no desenvolvimento de crianças pré-escolares. Licere. 2009; 12 (1): 1-21.

19. BRAGA MAM, GRACIANI Z. O brincar na rotina da criança com paralisia cerebral. Cadernos de Pós-Graduação em Distúrbios do Desenvolvimento. 2015; 15 (1): 41-49.

20. SIAULYS MOC. Brincar para todos. Brasília: Ministério da Educação, Secretaria de Educação Especial, 2005; 152 p.: il. color.

21. WAKSMAN RD, HARADA MJCS. Escolha de brinquedos seguros e o desenvolvimento infantil. Revista Paulista de Pediatria. 2005; 1 (23): 36-48.

22. ALEXANDER GM, WILCOX T, WOODS R. Sex Differences in Infants' Visual Interest in Toys. Archives Of Sexual Behavior. 2008; 38 (1): 427-433.

23. TODD BK, BARRY JA, THOMMESSEN SA. O. Preferences for 'Gender-typed' Toys in Boys and Girls Aged 9 to 32 Months. Infant and Child Development, 26: e 1986. doi: 10.1002/icd.1986.

Dados dos autores

BIANCA ALVES ALMOZARA BONTORIM

Graduanda em Fisioterapia na Universidade Metodista de Piracicaba. Piracicaba/SP - Brasil. babontorim@ unimep.br

\section{AUDREI FORTUNATO MIQUELOTE}

Doutoranda do programa de Pós-graduação em Ciências do Movimento Humano na Universidade Metodista de Piracicaba. Piracicaba/SP - Brasil. audreifortunato@gmail.com

\section{CASSIANA DO AMARAL COPPOLA}

Mestranda do programa de Pós-Graduação em Ciências do Movimento Humano na Universidade Metodista de Piracicaba. Piracicaba/SP - Brasil. cassicoppola@gmail.com

\section{ANDREA BARALDI CUNHA}

Doutora em Fisioterapia pela Universidade Federal de São Carlos. Piracicaba/SP - Brasil. andreabaraldi@ gmail.com

\section{GABRIELLA PADOVANI}

Graduanda em Fisioterapia na Universidade Metodista de Piracicaba. Piracicaba/SP - Brasil. gabriellapadovani4@gmail.com

VANESSA ARAÚJO SILVA

Graduanda em Fisioterapia na Universidade Metodista de Piracicaba. Piracicaba/SP - Brasil. vanessa.araujo10@hotmail.com

\section{DENISE CASTILHO CABRERA SANTOS}

Doutora em Ciências Médicas pela Universidade Estadual de Campinas. Professora orientadora no Programa de Pós-Graduação em Ciências do Movimento Humano da Universidade Metodista de Piracicaba. Piracicaba/ SP - Brasil. dcsantose@unimep.br

Submetido em: 31-10-2017

Aceito em; 6-11-2017 